
allemande

51-2| 2019

Les Humanités environnementales : circulations et renouvellement des savoirs en France et en Allemagne

\title{
Le cheval astronomique à la croisée des savoirs : une lecture des scènes de foire dans Woyzeck de Georg Büchner
}

Elisabeth Hamm

\section{OpenEdition}

\section{Journals}

Édition électronique

URL : https://journals.openedition.org/allemagne/1981

DOI : 10.4000/allemagne. 1981

ISSN : 2605-7913

Éditeur

Société d'études allemandes

Édition imprimée

Date de publication : 10 décembre 2019

Pagination : 357-370

ISSN : 0035-0974

Référence électronique

Elisabeth Hamm, "Le cheval astronomique à la croisée des savoirs : une lecture des scènes de foire dans Woyzeck de Georg Büchner », Revue d'Allemagne et des pays de langue allemande [En ligne], 51-2 I 2019, mis en ligne le 02 décembre 2020, consulté le 23 mai 2021. URL : http://

journals.openedition.org/allemagne/1981; DOI : https://doi.org/10.4000/allemagne.1981 


\section{Le cheval astronomique à la croisée des savoirs: une lecture des scènes de foire dans Woyzeck de Georg Büchner}

- Elisabeth Hamm*

\section{Introduction: Les études animales littéraires pour repenser la nature}

Au sein des études littéraires, les animaux sont devenus un objet de recherche à part entière. Les Cultural and Literary Animal Studies (CLAS) ${ }^{(1)}$ se penchent sur des textes dans lesquels l'animal joue un rôle central, des œuvres d'auteurs qui traitent l'animal d'une manière particulière, ou des auteurs qui ont fait des recherches en sciences ou en zoologie à côté de leur activité littéraire. Les CLAS questionnent le statut de l'animal en littérature et analysent ce que cela signifie pour l'auteur, l'époque, le genre, quand ces éléments sont étudiés à partir de l'animal. Le but des CLAS est de montrer en quoi les textes littéraires peuvent être considérés comme des supports de représentations animales dans leur contexte historique, mais aussi en quoi ils peuvent prendre aujourd'hui un autre sens. Si les animal studies font partie des enjeux des humanités environnementales, c'est avant tout parce que ces dernières s'intéressent particulièrement aux notions de nature et de culture et s'inscrivent dans un mouvement questionnant la manière dont on peut (re)penser la nature ${ }^{(2)}$. Les animaux vivent dans un environnement spécifique, les animal studies doivent ainsi inclure des perspectives et des méthodes des humanités environnementales ${ }^{(3)}$. À l'inverse, l'environnement est toujours peuplé d'animaux

* Doctorante en études germaniques à l'Université Sorbonne Nouvelle-Paris 3, en cotutelle avec l'Université de Wurtzbourg, hamm.elisabeth@wanadoo.fr.

1 L'introduction suivante offre une ouverture théorique sur les CLAS: Roland BorgARDs, «Introduction: Cultural and Literary Animal Studies», Journal for Literary Theory, 9/2 (2015), p. 155-160.

2 Sur le lien entre humanités environnementales et animal studies, voir Roland Borgards, "Animal Studies», in: Aurélie Choné, Isabelle Hajex et Philippe Hamman (éd.), Rethinking Nature: Challenging Disciplinary Boundaries, Londres/New York, Routledge, 2017, p. 221-232.

3 En France, on assiste ces dernières années à un essor de projets portant sur les humanités environnementales. Dans le Guide des Humanités environnementales, plusieurs chapitres sont d'ailleurs 
spécifiques, ce qui fait que les animal studies viennent compléter les humanités environnementales ${ }^{(4)}$. Ainsi ces dernières se construisent-elles comme des champs disciplinaires structurant et redéfinissant les disciplines elles-mêmes. Repenser l'animal, c'est donc aussi repenser l'humain et les sciences humaines.

\section{Étude d'un cas concret tiré de Woyzeck}

Avec les animal studies se développe un autre rapport à l'objet littéraire, ce qui permet de revisiter la manière dont on approche les textes, notamment les textes canoniques. Il s'agira de montrer concrètement comment les animal studies s'appliquent à un texte littéraire. Cet article se propose d'effectuer une micro-lecture des «scènes de foire» ("Jahrmarktszenen» ${ }^{(5)}$ ), c'est-à-dire des fragments H 1,1 et H 1,2 tirés de Woyzeck de Georg Büchner ${ }^{(6)}$. Il s'agit d'une pièce inachevée et fragmentaire écrite en 1837, dont l'ordre des scènes n'est pas établi ${ }^{(7)}$.

Georg Büchner est un écrivain et scientifique allemand ayant étudié la philosophie, soutenu une thèse de biologie et enseigné l'anatomie à l'université( ${ }^{(8)}$. Dans Woyzeck, le protagoniste, qui vit difficilement, sombre dans la folie: lorsqu'il soupçonne sa compagne d'adultère, il perd la raison et la tue brutalement. Dans Woyzeck, l'animal se substitue à l'homme: dans le fragment H3,1, «Der Hof des Professors » ${ }^{(9)}$, Woyzeck est traité comme le chat sur lequel il expérimente. De ce fait, Woyzeck se perçoit lui-même comme un animal soumis à ses besoins naturels, et c'est notamment l'une des raisons pour lesquelles il va sombrer dans la folie. La frontière entre animalité et humanité est donc floue dans Woyzeck. Dans les fragments qui nous intéressent, Woyzeck et Marie se

consacrés à la question animale: Aurélie Choné, Isabelle Hajek et Philippe Hamman (dir.), Guide des Humanités environnementales, Villeneuve d'Ascq, Presses universitaires du Septentrion, 2016.

4 L'entrée "Animal Studies" du Metzler Lexikon Literatur- und Kulturtheorie renvoie directement à l'entrée «Ecocriticism/Ökokritik». Les animal studies sont donc traitées dans le cadre de l'écocritique. Voir Ansgar NüNnING (éd.), Metzler Lexikon Literatur- und Kulturtheorie, Stuttgart/Weimar, Metzler, 2013 (5 éd.), p. 26.

5 Il s'agit du nom couramment donné à ce groupement de fragments, et non du titre donné explicitement par l'auteur.

6 Cet article s'appuie sur l'édition suivante du texte original de Woyzeck en allemand: Georg BüCHNER, Woyzeck. Sämtliche Werke und Schriften. Historisch-kritische Ausgabe mit Quellendokumentation und Kommentar (Marburger Ausgabe), vol. 7.2, par Burghard Dedner et Thomas Michael MAYer (éd.), Darmstadt, Wissenschaftliche Buchgesellschaft, 2005.

7 La pièce Woyzeck est caractérisée par Jean-Louis Besson et Jean Jourdheuil de "casse-tête littéraire et dramaturgique» dans la préface de leur traduction de la pièce. C'est à cette traduction que renvoie chaque citation du texte original. Georg Büchner, Woyzeck. Texte, manuscrits, source, trad. de JeanLouis Besson et Jean Jourdheuil, Paris, Théâtrales, 2004. Pour faciliter la lecture de l'article qui repose sur des remarques stylistiques, les citations restent dans l'allemand original dans le corps du texte et la traduction est mise en note.

8 Voir deux ouvrages de référence sur la vie et l'œuvre de Georg Büchner: Jan-Christoph HaUsCHILD, Georg Büchner. Verschwörung für die Gleichheit, Hambourg, Hoffmann und Campe, 2013; Jean-Louis Besson, Le Théâtre de Georg Büchner. Un jeu de masques, Belfort, Circé, 2002.

9 Woyzeck, p. 20. Certains éditeurs considèrent que cette scène n'est qu'une première ébauche et qu'elle ne doit donc pas être retenue dans la version définitive. Dans la traduction de Jean-Louis Besson et Jean Jourdheuil, "La cour du professeur» ne figure pas dans la version reconstituée, mais dans la traduction des manuscrits, p. 80 . 
trouvent dans une foire où un Annonceur tente d'attirer les passants à une représentation avec un cheval astronomique qui sait compter et lire l'heure. Marie veut voir le spectacle et entre dans la baraque avec Woyzeck ${ }^{(10)}$. À l'intérieur, le Bonimenteur tient un discours sur la nature et la raison dans lequel l'être humain et l'animal ne se distinguent plus. Cet exemple tiré d'un extrait d'une pièce de théâtre datant du début du XIX ${ }^{\mathrm{e}}$ siècle montre en quoi on peut observer à cette époque un tournant aussi bien au niveau de la conception de l'animalité que de sa représentation au théâtre. Un tout nouveau bestiaire apparaît sur les planches: l'animal revêt des rôles jusque-là inexplorés et sa vie, son langage et sa pensée sont mis en scène. C'est donc surtout au XIX ${ }^{\mathrm{e}}$ siècle que l'anthropocentrisme est remis en cause et que la question animale change radicalement d'orientation.

Il existe de nombreuses études et analyses sur les scènes de foire dans Woyzeck, d'une part ${ }^{(11)}$, et sur les animaux chez Büchner, d'autre part ${ }^{(12)}$, qui montrent notamment que, dans Woyzeck, les animaux revêtent des fonctions différentes. Mais on ne trouve pas encore vraiment de travail exhaustif sur le cheval dans ces scènes, il s'agit plutôt de l'étude du motif en tant que tel dans la pièce. Martin Wagner montre en quoi le cheval anthropomorphisé et le caractère interchangeable des rapports entre le cheval et l'être humain sont introduits dans les scènes de foire pour être repris ensuite en tant que motif structurant dans toute la pièce ${ }^{(13)}$. Le chien et le chat, deux animaux qui interviennent dans des scènes ultérieures ${ }^{(14)}$ et qui ont ceci en commun avec le cheval qu'ils ne se différencient plus de l'homme, réactivent alors structurellement l'inversion entre l'homme et l'animal introduite dans les scènes de foire et apparaissent comme des avatars du cheval. En s'appuyant sur des concepts de Judith Butler (to call someone a name, interpellation) ${ }^{(15)}$, Martin Wagner explique aussi comment ce motif montre que c'est la société qui transforme Woyzeck en animal.

Ainsi, la micro-lecture que se propose d'effectuer cet article sera centrée sur le rôle que joue le cheval, pour deux raisons. D'un côté, le cheval est un animal ayant un rapport étroit aux légendes et aux mythes germaniques ainsi qu'à la culture populaire et au folklore de cet espace, comme le montre Marc-André Wagner ${ }^{(16)}$. Cet animal a déjà été beaucoup étudié en tant que symbole littéraire ${ }^{(17)}$, notamment dans les contes,

10 Suivis du tambour-major et d'un sous-officier dans H2,3.

11 Roland Borgards, «Performing Species. Menschenpolitik und Tiertheorie im "Woyzeck" (H 1,1; H 1,2)», in: Georg Büchner Jahrbuch 13 (2013-2015), Berlin, De Gruyter, 2016, p. 257-274.

12 Roland Borgards, "Tiere», in: Roland Borgards, Harald Neumeyer (éd.), Georg Büchner. Epoche - Werk - Wirkung, Stuttgart, Metzler, 2009, p. 218-225.

13 Martin WAgner, «Büchner und Butler: Das Pferde-Narrativ in den Woyzeck-Entwürfen und die Handlungsfähigkeit des postsouveränen Subjekts», Monatshefte, Madison, University of Wisconsin Press, 104/4 (2012), p. 477-488.

14 Dans H4,9 («Woyzeck. Der Doktor»), Woyzeck devient un chien qui urine sur les murs. Dans H3,1 («Der Hof des Professors»), Woyzeck apparaît en tant qu'assistant d'un professeur qui fait une expérience avec un chat, mais quand le chat s'enfuit, c'est Woyzeck lui-même qui devient l'objet d'expérimentation.

15 Judith Butler, Le pouvoir des mots. Politique du performatif, Paris, Éditions Amsterdam, 2004.

16 Marc-André WAgner, Le cheval dans les croyances germaniques: paganisme, christianisme et traditions, Paris, Honoré Champion, 1974.

17 Pour cet aspect, se référer à l'article de Gertrud Maria Rösch, «Pferd», in: Günter Butzer, Joachim JАсов (éd.), Metzler Lexikon literarischer Symbole, Stuttgart/Weimar, Metzler, 2008, p. 274. 
ou comme référence à l'emblématique des $\mathrm{XVI}^{\mathrm{e}}$ et $\mathrm{XVII}{ }^{\mathrm{e}}$ siècles, car il révèle un rapport particulier entre chevalier et monture, qui se rapproche alors de celui entre le seigneur et ses sujets ${ }^{(18)}$. Il est aussi très présent dans le discours anatomique et philosophique de l'époque et permet donc d'interroger la spécificité de l'espace germanophone par rapport à la question animale. Le cheval astronomique qui nous intéresse est un cheval savant qui sait compter et lire l'heure, motif qui se développe surtout plus tard au cours du XIX ${ }^{\mathrm{e}}$ siècle, puis au début du XX $\mathrm{XX}^{\mathrm{e}}$ siècle, avec Hans le malin ${ }^{(19)}$ (Kluger Hans), mais qui témoigne déjà d'un intérêt pour le cheval comme élément de la culture et du folklore germanique, en tant que phénomène de foire. D’un autre côté, le cheval est un animal récurrent dans la pièce et ce n'est pas le seul lien que l'on peut faire entre le cheval et le folklore: la récurrence de ce motif dans Woyzeck et le fait qu'il apparaisse dans les chansons populaires citées par les personnages, dans le fragment $\mathrm{H} 4,2$ notamment ${ }^{(20)}$, montrent que le cheval est repris dans son rapport à la tradition. Dans le fragment H3,2, ce n'est pas non plus un hasard s'il est question d'un «Reuter» ${ }^{(21)}$, qui, tout en faisant allusion au champ lexical équestre ${ }^{(22)}$, fait référence à une sorte de petit gâteau ${ }^{(23)}$. Deux lignes plus loin, Woyzeck cite d'ailleurs une comptine pour enfants avec «Hop! Hop ! Roß» ${ }^{(24)}$, ce qui ancre encore plus le cheval en tant qu'animal dans la culture populaire allemande.

\section{Le cheval astronomique, vecteur d'un savoir culturel et littéraire sur l'équidé au début du XIX ${ }^{\mathrm{e}}$ siècle}

Le cheval astronomique présent dans Woyzeck s'inscrit dans l'imaginaire collectif de l'espace germanophone de l'époque. Il porte avec lui toute une série d'images et il est intéressant de voir comment Büchner les traite. Dans le fragment $\mathrm{H} 1,1$, le cheval astronomique est introduit sans didascalie et désigné d'emblée comme «astronomique» dans une structure passive («ist zu sehn das astronomische Pferd» ${ }^{(25)}$ ). Ce n’est pas le seul animal présent dans le premier fragment, il y a aussi des oiseaux («die feinen Kanaillevögele» ${ }^{(26)}$ ) - et plus loin un singe déguisé en soldat - mais l'accord du verbe, «ist» et non "sind», montre que l'attention est d'ores et déjà attirée sur le cheval. Il est aussi intéressant de remarquer que le cheval est déjà annoncé par «He!

18 Beate Ackermann-Arlt, Das Pferd und seine epische Funktion im mittelhochdeutschen "Prosa-Lancelot", Berlin, De Gruyter, 1990.

19 Vinciane Despret, Hans, le cheval qui savait compter, Paris, Les Empêcheurs de penser en rond, 2004.

20 Marie chante: "Hansel spann deine sechs Schimmel an / Gib ihn zu fresse auf's neu / Kein Haber fresse sie / Kein Wasser saufe sie / Lauter kühle Wein muß es sein Juchhe / Lauter kühle Wein muß es sein», Woyzeck, p. 23. Traduction: «Hansel, attelle six chevaux blancs / Donne-leur à manger promptement / L'avoine, ils n'en mangent pas / L'eau, ils n'en boivent pas / Ce qu'ils veulent, c'est du vin frais. Tralala / Ce qu'ils veulent, c'est du vin frais», p. 23.

21 Woyzeck, p. 21.

22 Reuter présente des sonorités semblables à Reiter, le cavalier.

23 «Reuter» a d'ailleurs été traduit par «cheval en pain d'épice» par Jean-Louis Besson et Jean Jourdheuil, p. 82 .

24 Woyzeck, p. 21. Trad.: «Hop! Hop! Cheval! Cheval!», p. 82.

25 Ibid., p. 3. Trad.: «ici vous verrez le cheval astronomique», p. 24.

26 Ibid., p. 3. Trad.: «les petits oiseaux des Canailleries», p. 24. 
Hopsa!» ${ }^{(27)}$, les premiers mots de Woyzeck dans le fragment $\mathrm{H} 2,3^{(28)}$, qui font implicitement référence à «Hop! Hop! Roß» que l'on retrouve à la fin de la pièce et qui évoquent acoustiquement un saut, et par extension un cheval. L'adjectif "astronomique", attribué au cheval, se fonde au demeurant sur un jeu de mot qui repose à la fois sur sa capacité prétendue à être versé dans l'astronomie ${ }^{(29)}$ - alors qu'en fait il sait compter et lire l'heure, mais aussi sur sa taille, sa prestance et son caractère imposant, ce qui évoque l'importance qui lui est accordée. La manière dont le cheval est introduit dans la scène montre par conséquent déjà bien qu'il s'agit là d'un animal particulier et qu'il doit être lu et vu dans ce qu'il a de spécifique. Nous avons donc ici affaire à un animal que nous ne pouvons pas appréhender seulement dans sa référence à un motif, mais aussi dans ce qu'il a de particulier. La question que l'on se pose est alors celle-ci: pourquoi justement a-t-on ici ce cheval en particulier? Pour répondre à cette question, il est important de comprendre en amont ce à quoi l'équidé fait référence, à quoi le lecteur ou le spectateur pense quand il lit son nom ou le voit.

Au début du XIX ${ }^{\mathrm{e}}$ siècle, le cheval jouait un rôle important, puisqu'il était omniprésent: en tant que moyen de transport, dans l'agriculture et dans l'armée ${ }^{(30)}$. Mais ici, il s'agit d'un cheval de foire, qui véhicule tout un lot d'images faisant référence à ce que l'on savait du cheval à cette époque. En effet, l'identification entre l'homme et l'animal qui a lieu dans la scène est introduite dans un cadre traditionnel et populaire: les chevaux qui savent compter appartiennent à l'inventaire de la foire, bien connu du public germanophone de l'époque. Il s'agit donc d'une scène courante, qui n'a rien d'inattendu pour le lecteur et le spectateur à l'époque de l'écriture de la pièce, et elle fonctionne comme une sorte de «citation» du quotidien. En effet, à la fin du XVIII ${ }^{\mathrm{e}}$ siècle, l'espace germanophone connaît une floraison de foires et de marchés, reflets de la vie rurale de l'époque. D'abord pensée comme un espace privilégié d'échanges et de commerce, la foire devient au début du XIX ${ }^{\mathrm{e}}$ siècle un lieu festif, consacré de plus en plus au divertissement. Cette transformation est le reflet du changement de régime politique et économique opéré dans la société( ${ }^{(31)}$. Le nombre de cirques, et plus particulièrement de cirques équestres mettant en scène des chevaux, dont le public est constitué essentiellement d'individus de la noblesse et de l'armée, augmente d'ailleurs drastiquement.

Cet engouement pour les animaux de foire fait écho à l'imaginaire autour du cheval que l'on trouve dans la littérature. Cet animal possède en effet bien souvent des propriétés magiques ou extraordinaires. Il revêt généralement des rôles positifs, comme dans les contes dans lesquels il est classique que le prince arrive sur son fidèle destrier pour sauver la princesse. Il s'agit là d'un topos de ce genre et le cheval apparaît

27 Ibid., p. 14. Trad.: «Hé! Hop-là!», p. 24.

28 Dans beaucoup d'éditions, H2,3 est placé juste avant $\mathrm{H}$ 1,1, car il semble s'agir de la reconstitution ayant le plus de sens pour l'évolution de l'action.

29 L'astronomie était une grande préoccupation de l'époque. En témoignent les innovations techniques et les débuts de la spectroscopie avec Joseph Fraunhofer, qui annoncent la naissance de l'astrophysique.

30 Éric Leroy du Cardonnoy et Céline Vial (dir.), Les chevaux: de l'imaginaire universel aux enjeux prospectifs pour les territoires, Caen, Presses universitaires de Caen, 2017.

31 Daniel Roche, La culture équestre de l'Occident, XVI -XIX $X^{e}$ siècle. L'ombre du cheval, t. I: Le cheval moteur. Essai sur l'utilité équestre, 2008, t. II : La gloire et la puissance. Essai sur la distinction équestre, 2011, t. III: Connaissance et passion, Paris, Fayard, 2015. 
donc souvent comme un adjuvant. Dans le conte «Das Schimmelchen», provenant d'Autriche, un tout petit cheval aide un pauvre garçon à conquérir la princesse grâce à ses prédictions. Ce motif repose sur une croyance populaire selon laquelle les chevaux possèdent la capacité de prédire l'avenir, ce qui montre que le cheval savant est perçu comme une figure plutôt positive dans l'imaginaire collectif. Cependant, le cheval prend parfois aussi une dimension négative et effrayante, comme dans les histoires de cavaliers fantômes ou de "Schimmelreiter» ${ }^{(32)}$, qui mettent en scène un cheval blanc ou un cavalier sur un cheval blanc et qui reposent sur une superstition populaire selon laquelle ce cheval annoncerait une tempête ${ }^{(33)}$. Le cheval et ses caractéristiques fantastiques ou merveilleuses sont donc très présents dans la littérature et la culture populaire de l'espace germanique, mais l'intérêt pour le cheval en littérature au début du XIX ${ }^{\mathrm{e}}$ siècle se montre aussi dans l'apparition de biographies de chevaux, comme Life of the Mecklenburg Mare Amante en 1804, Life of a Job Horse en 1807 et Life of a Worn-Out Hack en 1819. Ces biographies ne voient certes pas le jour au sein de l'aire germanophone, mais elles y ont été lues et alimentent le discours littéraire de l'époque autour du cheval dans le sens où ces textes attribuent des émotions et des capacités cognitives aux chevaux, tout en défendant un traitement humain pour ces animaux à travers des homologies inter-espèces. Le cheval astronomique dans les scènes de foire de Woyzeck n'est donc pas qu'un motif littéraire et un symbole. Il s'agit du reflet d'une construction culturelle autour du cheval, animal omniprésent dans l'imaginaire collectif de l'espace germanophone. Il porte en lui une série d'images et véhicule un savoir, ce qui influence la manière dont il est perçu par le lecteur ou le spectateur.

\section{Le cheval astronomique, reflet du discours scientifique, anatomique et philosophique de l'époque}

Le cheval astronomique de Büchner apparaît aussi comme le reflet des discours scientifique et philosophique de l'époque, qui sont indissociables l'un de l'autre et dans lesquels le cheval est omniprésent. Dans les fragments qui nous intéressent, l'homme et l'animal sont non seulement inversés, mais aussi interchangeables. Nous allons voir comment fonctionne ce renversement et comment il est mis en scène. Dans le premier fragment, le cheval n'est pas le seul animal présent. Dès le début, Büchner thématise l'opposition entre le monde des humains, caractérisé par une forme de memento mori («wir müssen alle sterben, das ist uns wohlbekannt! » ${ }^{(34)}$ ) et ses conditions pénibles, évoquées ironiquement par le «Welt! Schöne Welt!» ${ }^{(35)}$ dans la première réplique de Woyzeck, et le monde de la foire, tournant autour du cheval. L'équidé est présenté par un Annonceur (Ausrufer) qui cherche à attirer des spectateurs potentiels en l'exhibant comme un animal savant:

32 Ces légendes reposent sur une longue tradition orale à laquelle un intérêt tout particulier est montré au XIX ${ }^{\mathrm{e}}$ siècle. En témoigne la publication en 1838 de "Der gespenstige Reiter. Ein Reiseabenteuer» dans les Danziger Dampfboot, puis plus tard en 1888 la nouvelle L'Homme au cheval blanc (Der Schimmelreiter) de Theodor Storm. Voir «Der gespenstige Reiter. Ein Reiseabenteuer», in: Johann Joseph Christian PAPpe (éd.), Lesefrüchte vom Felde der neuesten Literatur des In- und Auslandes, Hambourg, 1838, vol. 2, p. 125-128; Theodor Sтокм, Sämtliche Werke, vol. 3, Essen, Mundus Verlag, 1999. 
le cheval, associé aux petits oiseaux, est «Liebling von allen Potentaten Europas» ${ }^{(36)}$, "Mitglied von allen gelehrten Societäten» ${ }^{(37)}$ et sait prédire l'avenir. Il est comparé à l'être humain et lui est même supérieur, puisque ce n'est pas un individu stupide, au contraire de beaucoup d'hommes («wie viele Personen, das verehrliche Publikum abgerechnet» ${ }^{(38)}$ ), notamment les soldats («unterst Stuf von menschliche Geschlecht» ${ }^{(39)}$ ). Il est également mis en avant comme un exemple du progrès de la civilisation ( Sehn Sie die Fortschritte der Civilisation» ${ }^{(40)}$ ). La confusion entre être humain et animal repose donc sur une anthropomorphisation, qui apparaît comme une stratégie de l'Annonceur pour attirer l'attention des passants en les flattant. La rhétorique de l'Annonceur repose sur un mélange d'éléments du langage quotidien, dialectaux et d'emprunts à des langues étrangères, ainsi que sur de nombreuses figures de répétition, qui fonctionnent également comme une référence indirecte au contexte militaire dans lequel se joue la pièce. L'Annonceur utilise en effet de nombreuses anaphores ("Meine Herren, meine Damen», «Meine Herren, meine Herren », «sehen sie [...] sehen sie», «hat [...] hat») et gradations («nix, gar nix »), ainsi que des allitérations et assonances («So bist baron » ${ }^{(41)}$, « Michel ist musikalisch» $\left.{ }^{(42)}\right)$, bien que ces dernières soient en lien avec le singe. Ce jeu avec les sonorités atteint son paroxysme dans les expressions «eine ganze vernünftige Viehigkeit » ${ }^{(43)}$ et «kein viehdummes Individuum » ${ }^{(44)}$, qui unissent au champ lexical de l'animal, dans une combinaison avec des oxymores et des néologismes, des caractéristiques habituellement attribuées aux humains comme la raison ou la notion d'individu. C'est donc avant tout à travers la rhétorique de l'Annonceur qu'opère l'inversion entre l'animal et l'être humain, en mettant en place une anthropomorphisation du cheval.

Dans le deuxième fragment, il ne s'agit plus seulement d'une inversion, mais aussi d'une véritable interchangeabilité. Le cheval est désormais le seul animal présent et on assiste, grâce à des répétitions, à un effet de gradation par rapport au premier fragment: le Bonimenteur (Marktschreier) reprend beaucoup d'éléments qui ont déjà été prononcés par l'Annonceur, comme les expressions «Mitglied von allen gelehrten Societäten» et "viehdummes Individuum», mais en les modifiant parfois. Le mot «Viehsionomik» ${ }^{(45)}$, par exemple, vient compléter l'expression «viehdummes Individuum » et contient tout le paradoxe de la situation de Woyzeck: la physionomie désigne en effet à la fois la nature intrinsèque d'une chose, mais aussi le fait que des traits physiques puissent être des indicateurs du caractère ou des émotions d'un individu. Chez Büchner, l'individu se retrouve alors déshumanisé et devient "viehdumm», aussi stupide qu'une bête. En interprétant le «vi» de «Individuum» en «Vieh», Büchner estompe la différence entre les humains et les animaux et gomme la prétendue

36 Ibid., p. 3. Trad.: "les préférés de tous les potentats d'Europe», p. 24.

37 Ibid., p. 3. Trad.: «membres de toutes les sociétés savantes», p. 24.

38 Ibid., p. 14. Trad.: «à l'exception de l'honoré public», p. 24.

39 Ibid., p. 14. Trad.: «le degré le plus bas du genre humain», p. 25.

40 Ibid., p. 14. Trad.: «Voyez les progrès de la civilisation», p. 25.

41 Ibid., p. 3. Trad.: «C'est bien, gentil», p. 24.

42 Ibid., p. 3. Trad.: «Michel est doué pour la musique», p. 24.

43 Ibid., p. 14. Trad.: «une animalité très raisonnable», p. 24.

44 Ibid., p. 14. Trad.: "ce n'est pas un individu bête comme un animal», p. 24.

45 Ibid., p. 3. Trad.: «bestiologie», p. 26. 
exception humaine. De plus, alors que dans le premier fragment on avait «wie viele Person ", le cheval lui-même devient une personne dans le deuxième fragment: «das ist eine Person ${ }^{(46)}$, ce qui souligne l'effet de gradation. Par deux fois, le Bonimenteur demande au cheval d'humilier la société humaine: la première fois, le cheval s'exécute et révèle l'étendue de son savoir. La deuxième fois, par contre, il refuse d'obéir en s'oubliant sur la scène, et c'est justement par ce refus qu'il montre qu'il est supérieur à l'homme: «So beschäme die société! Sehn sie das Vieh ist noch Natur unverdorbne Natur! Lernen Sie bey ihm ${ }^{(47)}$. Le cheval est donc ambivalent: d'un côté il est capable de se servir de sa raison d'une manière que l'homme ne peut égaler, et d'un autre côté il est avant tout nature, au-delà de toute considération culturelle. Les mots du Bonimenteur prennent dès lors une fonction performative, autrement dit l'interchangeabilité entre l'animal et l'être humain se produit quand il la formule: le cheval est maintenant apparenté à un humain ("ein verwandter Mensch» ${ }^{(48)}$ ), ce qui les met au même niveau. L'équidé présent dans la pièce est élevé au rang d'exemple pour tous les animaux, ce qui est confirmé par la manière dont il est désigné(49) : «dies Tier», " ein Vieh», "eine Bête», «das Pferd» (dans une didascalie) et «das Vieh». Nous sommes donc au départ en présence d'un pronom démonstratif, puis interviennent des articles indéfinis et finalement des articles définis. Ce phénomène souligne bien l'idée que l'on part d'un cheval particulier pour aller vers une généralisation et souligne en même temps le rôle que peuvent jouer les pronoms et les déterminants dans l'interprétation d'un texte s'appuyant sur les Cultural and Literary Animal Studies.

Nous avons donc affaire à un exemple littéraire d'inversion et d'interchangeabilité entre l'animal et l'être humain. Cet exemple est le reflet du discours scientifique, anatomique et philosophique de l'époque car cet équidé n'est en effet appréhendable que dans le contexte d'un débat naissant à l'époque autour de la frontière entre l'homme et l'animal. Plus précisément, ce contexte consiste dans le début d'une remise en question de l'anthropocentrisme. Au XIX ${ }^{\mathrm{e}}$ siècle, la découverte progressive de l'évolution des espèces vivantes aboutit à la réfutation progressive des conceptions venant notamment de la tradition judéo-chrétienne qui affirmaient que le monde avait été créé tel qu'il est encore aujourd'hui. Il y a d'ailleurs une allusion ironique à cette tradition dans le premier fragment, quand l'Annonceur désigne le singe comme «die Kreatur, wie sie Gott gemacht » ${ }^{(50)}$. L'évolution montre que l'humanité n'a pas toujours existé, qu'elle est le produit d'une série de transformations, et que les espèces ne sont pas immuables. La théorie de l'évolution de Darwin voit le jour en 1859, donc seulement après l'écriture de Woyzeck, mais on assiste déjà dans la première moitié du XIX ${ }^{\mathrm{e}}$ siècle à des remises en cause de la différence anthropologique ${ }^{(51)}$, comme en témoignent, par exemple, les travaux de Cuvier sur l'anatomie comparée à partir de 1800. Il s'agit de comparer

46 Ibid., p. 3. Trad.: «c'est une personne», p. 26.

47 Ibid., p. 3. Trad.: «Vas-y, fais honte à la société! Voyez, la bête: nature, nature non corrompue! Mettezvous à son école», p. 26.

48 Ibid., p. 3. Trad.: «un être humain en métamorphose», p. 26.

49 La traduction est fidèle à cette évolution, car le cheval est caractérisé dans l'ordre par «cet animal», «une bête», «un bestiau», et enfin «le cheval», «la bête».

50 Ibid., p. 3. Trad. : «la créature, telle que Dieu l’a faite», p. 26.

51 Quentin Deluermoz, François Jarrige, «Introduction. Écrire l'histoire avec les animaux", Revue d'histoire du XIX siècle, 54/1 (2017), p. 15-29. 
l'anatomie de différentes espèces (animales, végétales, fongiques) pour déterminer les processus adaptatifs de chacune d'entre elles à leur environnement, ainsi que d'opposer non seulement l'apparence mais aussi la structure des organes (histologie comparée) afin de distinguer les cas d'homologie des cas d'analogie évolutive. C'est donc une source importante de données servant principalement à l'étude de l'évolution du vivant.

Alors qu'aux $\mathrm{XVII}^{\mathrm{e}}$ et $\mathrm{XVIII}^{\mathrm{e}}$ siècles on assiste globalement à une radicalisation de la frontière entre l'homme et l'animal, Büchner s'intéresse à cette question dès ses premiers travaux, bien que sa conception se soit nuancée au fil du temps ${ }^{(52)}$. Il a en effet écrit une thèse de doctorat consacrée à l'anatomie du système nerveux du barbeau. Ce travail s'inspire à la fois des travaux de dissection et de la physiologie moderne du système nerveux, ce qui montre son intérêt pour le détail anatomique ainsi que pour l'exploration de l'intérieur du corps ${ }^{(53)}$. Dans son œuvre littéraire, et plus particulièrement dans les scènes de foire de Woyzeck, l'influence de ces centres d'intérêt est perceptible: les choses les plus simples s'inscrivent dans le cadre d'une remise en cause constante entre la nature et la culture. La dissection est certes une méthode pour analyser l'intérieur des corps, mais elle est aussi le moyen d'apporter à la littérature des caractéristiques propres à la science empirique à l'époque en pleine expansion, à savoir l'observation objective et la description clinique, qui doivent remplacer les constructions utopiques et idéalistes ${ }^{(54)}$. Dans les scènes qui sont l'objet de notre étude, l'analogie biologique entre l'être humain et l'animal devient donc le sujet d'un discours sur la nature contenant des implications morales. Entre l'homme et l'animal n'existe qu'une zone d'entre-deux dans laquelle ils sont interchangeables et Büchner apparaît alors comme un précurseur. Dans ces fragments, la problématisation de la frontière entre l'homme et l'animal, qui va de pair avec la remise en question de la différence anthropologique, est poussée à l'extrême.

Si le cheval astronomique dans Woyzeck, en ce qu'il problématise le bouleversement de la frontière entre l'être humain et l'animal, apparaît comme le reflet du discours scientifique et philosophique de l'époque à propos de la remise en question de l'anthropocentrisme, il met également en lumière un cas particulier au sein de ce discours, à savoir le cas de l'équidé, qui y est omniprésent. Le premier traité comprenant une étude approfondie du cheval paraît à Venise sous la plume de Carlo Ruini en 1598, qui consacre une première partie à une description anatomique précise et novatrice du cheval, artistiquement illustrée par des planches représentant des organes isolés ou des dissections complètes. La publication de ce premier traité d'anatomie consacré au cheval montre donc un chemin inverse à ce qui est habituel: au lieu de voir l'artiste puiser dans les acquis scientifiques un savoir utile à leur art, on imagine qu’il est probable que les artistes aient précédé les scientifiques dans l'étude

52 R. Borgards, «Performing Species» (note 11).

53 Laurence DAHAN-GAIDA, "Écrire avec les nerfs. Médecine et anatomie chez Georg Büchner», in: Laurence Talairach-Vilmas (dir.), Mécaniques du vivant: Savoir médical et représentations du corps humain (XVII $-X I X^{e}$ siècle), Actes du colloque Explora, 2-3 décembre 2011, mis en ligne en novembre 2012 sur le site Épistémocritique, epistemocritique.org/category/ouvrages-en-ligne/actesde-colloques/mecaniques-du-vivant-savoir-medical-et-representations-du-corps-humain-xviiexixe-siecle/.

54 Marion Schmaus, Psychosomatik. Literarische, philosophische und medizinische Geschichten zur Entstehung eines Diskurses (1778-1936), Tübingen, Niemeyer, 2009. 
de l'anatomie équine ${ }^{(55)}$. En 1766, George Stubbs publie The Anatomy of the Horse, including a particular description of the Bones, Cartilages, Muscles, Fascias, Ligaments, Nerves, Arteries, Veins and Glands, in Eighteen Tables, all done from Nature, by George Stubbs, Painter, un ouvrage qui regroupe un ensemble d'illustrations des dissections de chevaux qu'il avait menées lui-même. Cet ouvrage nous montre un nouvel exemple de transfert de l'art vers la science. Comme Ruini avait été sans cesse recopié, les gravures de Stubbs furent largement reprises et intégrées à des ouvrages de maréchalerie et des ouvrages scientifiques. Ces gravures, bien qu'imparfaites sur le plan de l'anatomie, furent réutilisées comme illustrations d'articles consacrés aux soins du cheval dans des encyclopédies britanniques et connurent un engouement marqué en Allemagne. Ernst Matthaei, artiste à Dresde, faisait remarquer en 1823 que ces planches, bien qu'imparfaites, étaient les meilleures du genre qui existaient à cette époque ${ }^{(56)}$. Du reste, d'Alton les avait reprises dans son histoire naturelle du cheval publiée à partir de 1810, et Schwab les reproduisait coloriées dans son Anatomische Abbildung und Beschreibung des Pferdekörpers, publiée à Leipzig en 1821.

Les progrès de l'anatomie et de la médecine au XVIII ${ }^{\mathrm{e}}$ et au XIX $\mathrm{X}^{\mathrm{e}}$ siècles permettent ainsi de passer de l'hippiatrie, désignant les soins qui incluaient des rituels magiques et des saignées, à une véritable médecine équine ${ }^{(57)}$. La première école vétérinaire ouvre ses portes à Lyon en 1761, puis en Allemagne à Göttingen en 1770, ainsi qu'à Hanovre en 1778 , à Dresde en 1780 et à Berlin et Munich en $1790^{(58)}$. Dans ces écoles vétérinaires, le cheval était le standard puisque l'anatomie et la physiologie par exemple se développèrent en prenant son corps pour modèle. La fin du XVIII ${ }^{\mathrm{e}}$ siècle a donc été une période particulièrement faste pour l'anatomie du cheval qui a apporté aux artistes $\mathrm{du} \mathrm{XIX}^{\mathrm{e}}$ siècle une matière première dont ils usèrent dans leurs représentations ${ }^{(59)}$. À partir de ceci put se développer l'hippologie, qui connut au début du XIX siècle en Allemagne un essor sans précédent. Nous pouvons citer à titre d'exemples les ouvrages de Franz Maximilian Friedrich Bouwinghausen von Wallmerode ${ }^{(60)}$ (1795), de Johann Georg Naumann ${ }^{(61)}$ (1800), de Joseph Wilhelm Eduard d'Alton, Naturgeschichte des Pferdes und seiner Rassen ${ }^{(62)}$ dans les années 1810, et enfin celui de Konrad Ludwig

55 Christophe Degueurce, «L'anatomie du cheval aux XVIII ${ }^{\mathrm{e}}$ et XIX ${ }^{\mathrm{e}}$ siècles: un outil pour mieux représenter le cheval», In Situ, 27 (2015), en ligne: journals.openedition.org/insitu/12058.

56 Burkhardt Wilhelm Seiler, Carl August Boettiger, Erklärungen der Muskeln und der Basreliefs an Ernst Matthaei's Pferde-Modelle, Dresde, Arnold, 1823.

57 Ulrich Raulff, Das letzte Jahrhundert der Pferde: Geschichte einer Trennung, Munich, C.H. Beck, 2015, p. 174.

58 Jens Oliver Kемpғ, Die Königliche Tierarzneischule in Berlin von Carl Gotthard Langhans. Eine baugeschichtliche Gebäudemonographie, Berlin, Mann, 2008.

59 C'est le cas de Géricault, comme le montrent les études de Lorenz Eitner, Géricault. His Life and Work, Londres, Orbis Publishing, 1982, et de Karin KüGler, Die Pferdedarstellungen Théodore Géricaults. Zur Entwicklung und Symbolik des Pferdemotivs in der Malerei der Neuzeit, mémoire de master, Université de Kiel, 1998.

60 Franz Maximilian Friedrich Bouwinghausen von Wallmerode, Taschenbuch auf das Jahr 1792 für Pferdeliebhaber, Reuter, Pferdezüchter, Pferdeärzte und Vorgesetzte großer Marställe, Tübingen, Cottaische Buchhandlung, 1795.

61 Johann Georg Naumann, Ueber die vorzüglichsten Theile der Pferdewissenschaft. Ein Handbuch für Offiziere, Bereiter und Oekonomen, Berlin, Himburg, 1800.

62 Joseph Wilhelm Eduard d'Alton, Naturgeschichte des Pferdes und seiner Rassen, Weimar, Landes Industrie Comptoir, 1810-1816. 
Schwab, professeur de médecine vétérinaire à Munich, en $1820^{(63)}$. Ces progrès en matière de sciences naturelles et d'anatomie montrent donc l'importance que jouait le cheval dans le discours de l'époque et les enjeux qui se nouent autour de lui. Ce n'est donc pas un hasard si Büchner décide de porter précisément cet animal sur les planches: le cheval astronomique incarne ce savoir et représente un exemple littéraire illustrant les travaux des scientifiques de l'époque.

\section{Le cheval astronomique sur la scène du savoir}

Le cheval présent dans les scènes de foire est indissociable du patrimoine qu'il porte en lui, mais l'analyse des fragments conduit également à se demander comment le texte produit de la théâtralité, et quel rôle joue précisément ce cheval dans la théâtralité. Tout d'abord, il apparaît que la rhétorique de l'Annonceur repose sur le champ lexical de la représentation théâtrale. Les passants sont hélés à plusieurs reprises («Meine Herren, meine Damen " ${ }^{(64)}$ ) et sont d'emblée considérés comme un public («das verehrliche Publikum»). Il est également question du début du spectacle (« es wird sein, die Rapräsentation, das commencement vom commencement wird sogleich nehm sein Anfang» ${ }^{(65)}$ ). Le cheval est mis en scène, mais c'est en fait la mise en scène qui est elle-même mise en scène, à travers une mise en abyme de la représentation. Le sujet de la scène n'est donc pas seulement le cheval, mais aussi la mise en scène du cheval. Le «commencement» dont il est question dans le premier fragment se réfère par ailleurs logiquement au début de la représentation dans le deuxième fragment, mais aussi au commencement de la pièce elle-même. Il est à cet égard important de préciser qu'il s'agit des fragments que Büchner avait d'abord pensés comme exposition pour sa pièce, ce qui souligne la particularité de leur fonction dramatique. En effet, en plus d'introduire le phénomène de théâtre dans le théâtre que l'on retrouve dans toute l'œuvre, les scènes de foire introduisent aussi l'atmosphère de la pièce et mettent en place le conflit dramatique, puisque c'est parce que Marie veut voir le cheval astronomique qu'elle fait la connaissance du Tambour-major, ce qui sera un prétexte et un déclencheur de la folie de Woyzeck, par la jalousie que la situation va susciter en lui. Autour du cheval astronomique se nouent donc les enjeux de l'exposition de la pièce, il est un moteur de l'action.

Le cheval astronomique possède également une dimension sensible: il est perçu à travers deux sens, la vue et l'ouïe (indirectement, à travers la voix de l'Annonceur puis celle du Bonimenteur), comme une attraction. Certaines parties de son corps sont évoquées: sa queue, son arrière-train, ses sabots, sa tête. Les doigts sont eux aussi mentionnés, justement car il n'en a pas, au contraire des humains, alors qu'il sait malgré tout compter. Pour le lecteur et le spectateur, le cheval est donc avant tout une entité physique. C'est la dimension sensible et la corporalité du cheval qui font que le gommage de la différence entre l'animal et l'être humain confère à la scène une dimension comique. La théâtralisation des rapports entre l'homme et l'animal ainsi que la manière dont le cheval est perçu produisent un effet comique sur le lecteur ou

63 Konrad Ludwig Sснwaв, Anatomische Abbildungen des Pferdekörpers, Munich, Kunst-Anstalt, 1820.

64 Woyzeck, p. 3 et p. 14.

65 Ibid., p. 14. Trad.: «Et tout de suite, la représentation, le commencement du commencement va débuter sur-le-champ», p. 24. 
le spectateur. Il s'agit là d'un rire grotesque, caractérisé par le goût du bizarre, de la déformation ici corporelle: le rire se transforme en grimace, car il est inséparable d'un certain malaise. La performance du cheval astronomique conduit donc à la fois au rire mais aussi à une forme de gêne. L'équidé, en tant qu'objet dramatique et par la manière dont il est théâtralisé, véhicule alors un savoir sur le théâtre et les animaux de scène.

Théâtre, savoir et animaux sont en effet intimement liés. Au moment de l'écriture de Woyzeck, les spectacles mettant en scène des animaux savants commencent à être de plus en plus nombreux, ce qui reflète la préoccupation de l'époque au sujet de l'intelligence animale: alors que l'on pensait que le comportement animal était uniquement dirigé par l'instinct, un débat entre instinct et intelligence s'installe et de nombreux scientifiques s'attellent à prouver que l'intelligence animale est bien supérieure à ce qu'imaginent les hommes ${ }^{(66)}$. Cet essor voit son paroxysme avec Hans le malin. À l'époque de Hans, d'autres animaux présentés comme "intelligents» sont exhibés au public dans le cadre de démonstrations et de spectacles. C'est le cas notamment de Rosa, la jument du musichall de Berlin, qui réalise des performances similaires à celles de Hans. On peut aussi citer un chien particulièrement intelligent à Utrecht, un cochon qui lit à Londres, ou encore Lady Wonder, la jument qui peut converser, mais aussi prévoir l'avenir et donner des conseils financiers. Le cheval astronomique présent dans Woyzeck fait par conséquent écho à tout un débat sur les animaux savants et au développement des spectacles proposant des performances animales. Le cheval astronomique, dans ce contexte de développement de la culture du divertissement et de progrès sur la connaissance de l'intelligence animale, préfigure les animaux stars qui viennent d'être évoqués.

L'histoire des chevaux de scène est également marquée par l'hippodrame ${ }^{(67)}$. Aux $\mathrm{XVIII}^{\mathrm{e}}$ et $\mathrm{XIX}^{\mathrm{e}}$ siècles, des scènes avec des chevaux ont été représentées au théâtre afin d'accentuer leur caractère sensationnel (dans les scènes de combat par exemple), mêlant ainsi théâtre et cirque, avec la voltige notamment ${ }^{(68)}$. Le Theater an der Wien, l'un des théâtres les plus en vogue au début des années 1800 dans l'espace germanophone, a proposé beaucoup de spectacles avec des chevaux dans son répertoire. Nous avons par ailleurs vu que Büchner portait un grand intérêt à la dissection, et qu'elle est étroitement liée à son écriture théâtrale. Il existe un lien étroit entre théâtre et dissection. À partir du $\mathrm{XVI}^{\mathrm{e}}$ siècle voient en effet le jour des édifices spécialisés où l'on procédait à des dissections en public, les théâtres anatomiques. Lanatomie fait donc partie intégrante de l'histoire de la scène. Les dissections publiques sont une mise en scène du dispositif anatomique, où l'on rend visibles les voies de la connaissance, l'objet à connaître et les protagonistes de l'entreprise de connaissance. À Berlin, l'école vétérinaire ouvre ses portes en 1790, et avec elle le Tieranatomisches Theater Berlin ${ }^{(69)}$, où des animaux étaient disséqués en public. Le cheval astronomique de Büchner apparaît comme le reflet de l'histoire culturelle de l'animal et plus particulièrement du cheval de scène, qui n'est alors plus

66 Deluermoz/JarRige, «Introduction. Écrire l'histoire avec les animaux» (note 51).

67 Arthur Hartley SAxon, Enter Foot and Horse: A History of Hippodrama in England and France, New Haven, Yale University Press, 1968.

68 Caroline Hodak, "Créer du sensationnel. Spirales des effets et réalisme au sein du théâtre équestre vers 1800 », Terrain. Anthropologie et sciences humaines, 46 (2006), p. 49-67.

69 Ses noms officiels sont Anatomisches Theater der Tierarzneischule ou Tieranatomisches Theater der Humboldt-Universität zu Berlin. 
seulement une scène de théâtre, mais aussi une scène du savoir. Si c'est précisément le cheval et pas un autre animal qui joue ce rôle dans la théorie et la pratique théâtrale, c'est, d'un côté, par un effet de citation de ce patrimoine culturel et historique, d'un autre côté, ce rôle est aussi lié au cheval en soi, qui, par ses caractéristiques propres, peut être considéré comme l'animal par excellence permettant de véhiculer le savoir.

\section{Conclusion}

L'exemple du cheval astronomique s'intègre dans les mécanismes d'une représentation à la fois scientifique et esthétique et pointe ainsi du doigt la spécificité de l'espace germanophone quant à la question de l'animal, puisque cet équidé porte en lui un patrimoine historique et culturel qui correspond à l'horizon d'attente d'un lecteur ou spectateur germanophone. Le cheval astronomique ne peut alors être compris que dans le contexte du discours de l'époque puisqu'autour du cheval se nouent plusieurs enjeux: animal proche de l'homme, on le retrouve aussi bien dans les foires que dans les travaux anatomiques, philosophiques et dans les théâtres. Il apparaît aussi comme le paradigme de la remise en question entre l'être humain et l'animal et même comme l'animal le mieux placé pour effectuer une circulation de savoirs entre l'art et les sciences naturelles, et inversement. Cette circulation permet de traverser les âges et de transférer au lecteur actuel des éléments de l'histoire culturelle du cheval, mais aussi un savoir sur le rapport entre l'homme et l'animal et les équidés dans le contexte du discours scientifique au moment de l'écriture de la pièce, ainsi qu'une certaine conception du cheval au théâtre.

Les Cultural and Literary Animal Studies offrent donc une nouvelle interprétation d'un texte littéraire en ce qu'elles révèlent ce qui se passe quand le cheval n'est plus compris qu'en tant que symbole ou motif pur. En ceci, elles influencent donc la manière dont on approche un texte littéraire et dont l'objet littéraire en soi peut être perçu, en répondant à la question: comment la littérature met-elle en scène les animaux? Elles deviennent alors structurantes et redéfinissent les disciplines et les études littéraires elles-mêmes: l'approche du texte de théâtre est renouvelée lorsque les animaux sont compris en tant qu'objets dramatiques. Chez Büchner, on observe également un mouvement de transfert: d'un côté, le cheval offre une clé pour l'interprétation de la scène, et, de l'autre, c'est la micro-lecture de la scène qui permet d'accéder à l'histoire culturelle du cheval ${ }^{(70)}$. C'est pour cette raison que le cheval doit être vu, comme Marie le dit à la fin de H 1,2: la scène apporte non seulement une représentation de l'animal, mais aussi un savoir sur le vivant, puisque le passage peut être lu en tant que texte porteur d'informations relevant des sciences naturelles. Nicolas Pethes conclut son article sur l'expérimentation humaine dans Woyzeck ${ }^{(71)}$ en définissant la pièce comme

70 C'est d'ailleurs l'une des réussites des humanités environnementales et des animal studies plus particulièrement que d'apporter un nouvel éclairage sur le lien entre littérature et histoire. Les textes littéraires, puisqu'ils détiennent un savoir sur l'animal, permettent à l'historien de les utiliser comme des sources et de faire de l'histoire du point de vue de l'animal. C'est dans cette perspective que travaille l'historien Éric Baratay, Biographies animales. Des vies retrouvées, Paris, Seuil, 2017.

71 Nicolas Pethes, «"Viehdummes Individuum”, "Unsterblichste Experimente”. Elements for a Cultural History of Human Experimentation in Georg Büchner's Dramatic Case Study "Woyzeck” ", in: Monatshefte, Madison, University of Wisconsin Press, 98/1 (2006), p. 68-82. 
un exemple d'«écriture de la connaissance» («writing of knowledge»). Avec le cheval astronomique, nous voyons donc que c'est également le cas à une petite échelle.

L'étude des procédés stylistiques mis en œuvre, bien que non-exhaustive, a montré en quoi les textes littéraires et plus particulièrement les textes de théâtre représentant des animaux pensés à l'époque comme effets de discours peuvent contribuer à mettre en évidence une véritable dramaturgie animale et à voir la scène comme un espace de connaissance. La micro-lecture de ces fragments de Woyzeck dans la perspective des Cultural and Literary Animal Studies permet alors de voir en quoi on assiste à une circulation et à un renouvellement des savoirs sur la nature, et montre aussi ce que les CLAS peuvent apporter aux humanités environnementales.

\section{Résumé}

Le présent article se propose d'effectuer une microlecture des "scènes de foire" tirées de Woyzeck de Georg Büchner, centrée sur le rôle que joue le cheval, afin de montrer concrètement en quoi les animal studies renouvellent l'approche du texte littéraire. Le cheval présent dans les fragments étudiés apparaît en effet comme le vecteur d'un savoir culturel et littéraire sur l'équidé au début du XIX $X^{e}$ siècle, et plus particulièrement du cheval de scène. Il est aussi le reflet du discours scientifique, anatomique et philosophique de l'époque. En offrant une nouvelle interprétation à un texte, les études animales littéraires révèlent ce qui se passe quand le cheval n'est plus compris qu'en tant que symbole ou motif pur. En ceci, elles influencent donc la manière dont l'objet littéraire et l'objet dramatique en soi peuvent être perçus, et répondent à la question: comment la littérature met-elle en scène les animaux? Elles deviennent alors structurantes et redéfinissent les disciplines des sciences humaines et les études littéraires elles-mêmes.

\section{Zusammenfassung}

Der vorliegende Artikel soll eine sich auf die Rolle des Pferdes stützende Mikrolektüre der "Jahrmarktszenen“ aus Georg Büchners Woyzeck vorschlagen. Hier wird gezeigt, inwiefern die Animal Studies die Art und Weise beeinflussen, wie man an einen literatischen Text herangeht. Das Pferd, das sich in den betreffenden Fragmenten befindet, erscheint als Träger eines kulturellen und literarischen Wissens über Pferde, und insbesondere Bühnenpferde, am Anfang des 19. Jahrhunderts. Es spiegelt auch den damaligen wissenschaftlichen, anatomischen und philosophischen Diskurs wider. Indem die Cultural and Literary Animal Studies eine neue Interpretation des Textes anbieten, bringen sie ans Licht, was vorkommt, wenn das Pferd nicht nur als Symbol oder als reines Motiv verstanden wird. Sie beeinflussen also die Art und Weise, wie der literarische bzw. theatralische Gegenstand verstanden werden kann, und geben eine Antwort auf die Frage, wie Literatur Tiere inszeniert. Deshalb können sie die Geistes- und Literaturwissenschaften selber strukturieren und neu definieren. 